Helmut Molly

\title{
Tysk. Et sprog - Fire stater - Fire kulturer?
}

8 forelæsninger holdt på Københavns Universitet. Udg. af SvenAage Jørgensen. Kopenhagen, München: Wilhelm Fink 1989. 168 s. (Text \& Kontext Sonderreihe Bd. 27).

Im Herbst 1988 fanden an der Universität in Kopenhagen eine Reihe von Veranstaltungen statt, auf denen man sich unter dem Hauptthema "Deutsch - eine Sprache - vier Kulturen?"1 mit Geschichte, Kultur und Literatur der damals vier deutschsprachigen Staaten beschäftigte. Für den vorliegenden Band wurden acht Vorlesungen ausgewählt, die "einen Eindruck von der Breite des Themas und seiner Komplexität vermitteln" sollen (S. 8). Niemand konnte 1988 voraussehen, wie schnell aus den vier Staaten drei werden würden, aber trotz des überholten Titels behält das Buch seine Bedeutung, weil es vor allem zeigt, auf welche Weise man sich in Kopenhagen mit dem Nachbarn im Süden auseinandersetzt: über die Hälfte der Beiträge befassen sich mit Deutschland in seinen wechselnden Erscheinungsformen.

Vibeke Winge untersucht in ihrer Vorlesung “Als Vimmelskaftet Tyskemannegade hieß" 2, wann und wo größere deutsche Einwanderergruppen nach Dänemark gekommen sind und welche Folgen das für Kultur und Sprache der Dänen gehabt hat. Sie findet die ersten Spuren einer Einwanderung bereits in lateinischen Quellen aus dem 13. Jahrhundert, und schon um 1300 sind in den größeren Hafenstädten Dänemarks regelrechte deutsche Kolonien anzutreffen: Kaufleute und Handwerker, die in diesen Städten zur politischen und wirtschaftlichen Führungsschicht gehörten. Außerdem waren Königshaus und Adel deutsch oder deutschsprachig, d.h. man beherrschte das Niederdeutsche, die lingua franca im gesamten Ostseebereich der Hanse. Von dieser Zeit an herrschte mehrere Jahrhunderte lang in Kopenhagen und in einigen anderen Städten eine Zweisprachigkeit, woran sich auch im 16. Jahrhundert, als das Niederdeutsche entsprechend der Entwicklung in Deutschland vom Hochdeutschen abgelöst

\footnotetext{
${ }^{1}$ Das Thema sowie die im Folgenden zitierten Stellen habe ich übersetzt.

2 Vimmelskaftet ist eine Straße im Zentrum Kopenhagens, Tyskemannegade bedeutet in wörtlicher Übersetzung "Deutschmännerstraße".
} 
wurde, nichts Wesentliches änderte. Erst im 19. Jahrhundert mit seinen nationalistischen Bewegungen war dann für die bis dahin selbstverständliche Zweisprachigkeit kein Platz mehr. Der breit angelegte Beitrag bietet eine Reihe illustrativer Zitate aus zeitgenössischen Quellen, demgegenüber kommen die sprachlichen Folgen der Einwanderung ein wenig zu kurz.

Dieter Lohmeier beschäftigt sich mit dem bis heute umstrittendsten Zeitabschnitt des 19. Jahrhunderts: "Der Kampf um Schleswig zwischen 1848 und 1864 aus der Sicht Schleswig-Holsteins und Preußens: Theodor Storm und Theodor Fontane.” Theodor Storm, Schleswig-Holsteiner von Geburt und aus Überzeugung, ergreift u.a. mit seinen politischen Gedichten für die Sache seiner Landsleute gegen die Dänen Partei. Storms Haltung läßt sich nach Lohmeiers Auffassung am ehesten mit dem Begriff des "politischen Glaubens" charakterisieren. Dieser von Nipperdey ${ }^{3}$ entlehnte Begriff versucht ein für das 19. Jahrhundert typisches Phänomen zu fassen, wo sich der Einzelne nach den Umwälzungen im Gefolge der Französischen Revolution nicht mehr hauptsächlich als Angehöriger einer hierarchisch strukturierten Gemeinschaft fühlt, sondern als Glied einer Gruppe von Gleichgesinnten in der Art einer Gemeinde. Diese Glaubenshaltung findet ihren Ausdruck nicht nur in Storms emotional geprägter Parteinahme, sondern auch in seiner Blindheit gegenüber den machtpolitischen Verhältnissen. Lohmeier weist darauf hin, daß diese Haltung für die Nationalliberalen auf beiden Seiten charakteristisch ist.

Bei Fontane stützt sich Lohmeier auf dessen Buch 'Der Schleswig-Holsteinische Krieg im Jahre 1864' (Berlin 1866) sowie auf den teils in Schleswig, teils in Kopenhagen spielenden Roman 'Unwiederbringlich' aus dem Jahre 1891. Er macht deutlich, daß für Fontane die Herzogtümer, die dieser eher als Teil der dänischen Welt betrachtet, nur eine Zukunft haben, wenn sie an Preußen kommen, denn dem preußischen Staat gehört die Zukunft - eine Beurteilung, die Storm nicht teilte. Es gelingt Lohmeier, die Unterschiedlichkeit der zeittypischen Haltungen Storms und Fontanes herauszuarbeiten, wobei freilich der Standort Fontanes etwas unscharf bleibt.

In seinem Beitrag "Hauptzüge der deutschen Geschichte nach 1870" geht es Per Øhrgaard vor allem darum, Entwicklungen und Zusammenhänge aufzuzeigen, die zu einem besseren Verständnis auch der Verschiebungen und Verwerfungen im Verlauf der wechselvollen Geschichte seit

\footnotetext{
3 Nipperdey, Thomas: Deutsche Geschichte 1800-1866. München 1983.
} 
der Einigung Deutschlands 1871 beitragen können. Daneben scheint an mehreren Stellen seine Absicht durch, mit Hilfe einer differenzierenden und relativierenden Sichtweise gängige Klischeevorstellungen der dänischen Zuhörer/Leser von den Deutschen und ihrer Geschichte zurechtzurücken.

Natürlich ist bei einer so knappen Darstellung die Verteilung der Schwerpunkte immer problematisch, insgesamt aber wirkt der Überblick relativ ausgewogen. An einigen Stellen allerdings meldet sich Widerspruch: So erhält der Leser gleich zu Anfang ein schiefes Bild von den Ereignissen anläßlich der Reichsgründung: Nicht Bismarck rief am 18. Januar 1871 Wilhelm I. zum Kaiser aus (so S. 43), sondern der Großherzog Friedrich von Baden; das fand nicht im Rahmen einer "Krönungszeremonie" (S. 43) statt, sondern es handelte sich um eine Kaiserproklamation - wer hätte auch den preußischen König zum "Deutschen Kaiser" (nicht "Kaiser des Deutschen Reiches" (S. 43)) krönen sollen? 1834 geschah nicht "die Gründung des Norddeutschen Zollvereins unter Preußens Leitung” (S. 44) - nach Verhandlungen zwischen norddeutschen und süddeutschen Staaten, die sich jeweils zu Zollvereinen zusammengeschlossen hatten, kam es zur Gründung des Deutschen Zollvereins, in dem Preußen eine Vormachtstellung hatte. S. 49 behauptet der Autor, daß "Deutschland allerdings nie [Reparationen nach dem 1. Weltkrieg] bezahlt hat" - man ist sich in der Forschung zwar nicht einig darüber, wieviel tatsächlich bezahlt wurde, aber daß Deutschland Zahlungen geleistet und Sachlieferungen erbracht hat, ist unbestritten, beispielsweise waren Rückstände in Holz- und Kohlelieferungen im Jahre 1923 für Frankreich der Anlaß zur Besetzung des Ruhrgebietes. Bei der Darstellung der Gründe für das Scheitern der Weimarer Republik tritt der personelle Aspekt zu sehr in den Hintergrund. Die Formel von Weimar als einer Demokratie ohne Demokraten bezieht sich ja nicht nur auf die Wahlbevölkerung, sondern auch auf die führenden Personen, allen voran den Reichspräsidenten von Hindenburg, der entscheidend dazu beitrug, daß sich die Demokratie von 1930 an in ein autoritäres Präsidialsystem verwandelte. Ähnliches gilt, wenn der Autor die bis 1944 anhaltende Steigerung der Rüstungsproduktion erklärt, wobei er den Anteil des damaligen Rüstungsministers Speer übersieht.

Die (Drei-)Teilung Deutschlands nach dem 2. Weltkrieg läßt sich ohne Berücksichtigung der Westverschiebung Polens durch Stalin und der damit verbundenen gigantischen Bevölkerungsbewegungen kaum angemessen darstellen. Was die BRD betrifft, sei nur auf ein Mißverständnis hingewiesen, das man oft in dänischen Darstellungen findet: Der Bundes- 
rat ist keine eigentliche "Zweite Kammer" (S. 65), die - wie z.B. der USSenat - aus vom Volk gewählten Vertretern der Bundesländer besteht, sondern ein ständiges Organ, das sich aus delegierten Vertretern der einzelnen Landesregierungen zusammensetzt.

Bei der Darstellung der DDR fällt eine gewisse Zurückhaltung auf, die den tatsächlichen Verhältnissen nicht immer gerecht wird. So ist der Bau der Berliner Mauer 1961 nicht primär Ausdruck dafür, daß man "öffentlich einräumt, die Konkurrenz mit dem Westen nicht geschafft zu haben" (S. 69) - das hat die DDR-Bevölkerung etwas anders gesehen. Der Abstand zum Niveau der Weltwirtschaft läßt sich nicht damit erklären, daß Innovationen in der DDR schwer durchzusetzen waren (vgl. S. 69), da gab es schwerwiegendere Gründe, die mit dem damals dort real existierenden Wirtschaftssystem zusammenhängen. Und die Bevölkerung war auch nicht verbittert darüber, daß es nur nach besonderer Erlaubnis möglich war zu reisen (vgl. S. 70) - es war für die Meisten eben überhaupt nicht möglich, was dann im Herbst 1989 eine gewisse Rolle gespielt hat. Vielleicht liegt es an dieser Sichtweise, daß der Autor mit seiner Prognose über die lange Zukunft der DDR (vgl. S. 70) nicht recht behalten hat. Aber diese Einwände können den Eindruck eines kenntnisreichen Beitrags nicht wesentlich beeinträchtigen, der auf knappem Raum der Komplexität des Themas auf bewundernswerte Weise Rechnung trägt.

Karl Christian Lammers schildert in seinem Beitrag mit dem provozierenden Titel "Österreichs Beitrag zur neueren Geschichte Deutschlands (Adolf Hitler und der Nationalsozialismus)" die Rolle Österreichs während der Entstehung und der Herrschaft des Nationalsozialismus. Er sieht Österreich nicht als bloßen Teil der deutschen Geschichte bis 1945, sondern als Akteur, der für den Verlauf dieser Geschichte mitverantwortlich zu machen ist. Seine Darstellung, die im Wesentlichen auf Gerhard Botz $^{4}$ fußt, widerspricht damit der in der Moskauer Erklärung von 1943 formulierten Auffassung der Alliierten, daß Österreich das erste Opfer der Aggression Hitlers gewesen sei. Diese Opferthese als "Lebenslüge" der Österreicher (S. 85) hat wesentlich dazu beigetragen, daß die Beschäftigung mit der eigenen Vergangenheit zunächst verdrängt werden konnte und eigentlich erst anläßlich des "Falles Waldheim" im Jahre 1986 einsetzte.

Birgit S. Nielsen beschreibt in ihrem Beitrag "Deutsche Schriftsteller auf der Flucht vor Hitler" den kürzeren oder längeren Aufenthalt von

\footnotetext{
4 Botz, Gerhard: Österreich und die NS-Vergangenheit. In: Diner, Dan (Hrsg.): Ist der Nationalsozialismus Geschichte? Frankfurt 1987.
} 
rund zehn deutschen Autoren in Dänemark, von denen Bertolt Brecht, Hans Henny Jahnn und Walter Kolbenhoff die bekanntesten sind. Trotz einer eher restriktiven Flüchtlingspolitik Dänemarks wurde ihre Flucht durch die großzügige Hilfe von Privatpersonen und privaten Hilfskomitees ermöglicht, wobei die bedeutende Rolle der dänischen Autorin Karin Michaëlis und ihres Freundeskreises gewürdigt wird.

In seinem anderen Beitrag - "Christa Wolf und die DDR: die Entwicklung einer Gesellschaft im Spiegel eines Werkes” - geht es Per Øhrgaard nicht darum, die Entwicklung der DDR-Gesellschaft anhand der Werke Christa Wolfs darzulegen, vielmehr liest er ihre Bücher als einen Kommentar zu zentralen Fragen der Zeit. Trotz dieser verengten Sichtweise, auf die der Autor ausdrücklich aufmerksam macht, rücken wesentliche Teile des Werks der engagierten Zeitgenossin in den Blick, von 'Der geteilte Himmel' (1963) bis zu 'Störfall' (1986). Die dabei zu beobachtende Bewegung von der Situation im geteilten Deutschland hin zu Fragen von globaler Relevanz ist in der Entwicklung des Werkes von Christa Wolf begründet. Gleichzeitig macht Øhrgaard auf eine zunehmende Skepsis der Autorin aufmerksam, nicht gegenüber dem Sozialismus als Entwurf, sondern gegenüber der Art, wie die DDR ihn verwirklicht. Ob es allerdings berechtigt ist, Christa Wolf deshalb zu "einer Art moralische Instanz" zu machen, "die sich am ehesten mit Heinrich Böll vergleichen läßt" (S. 30), darf bezweifelt werden, dazu war der Einfluß beider Autoren auf ihre Gesellschaft (und die Möglichkeit dazu) zu unvergleichbar ${ }^{5}$. Die Darstellung der DDR-Verhältnisse bewegt sich stellenweise in erstaunlicher Nähe zu (ehemals) offiziellen Lesarten, beispielsweise wird 'Der geteilte Himmel' als "ein Stück DDR-Nationalliteratur Anno 1963” (S. 26) bezeichnet, die wirtschaftlichen Schwierigkeiten der Anfangsphase werden zum Teil auf "westliche Sabotage" (S. 27) zurückgeführt, oder der Aufstand im Juni 1953 findet dadurch sein Ende, daß "russisches Militär eingriff, um Ruhe und Ordnung aufrechtzuerhalten" (S. 27). Øhrgaard verdeutlicht eindrucksvoll die innere Konsequenz in der Entwicklung des Werkes von Christa Wolf, indem er die Verbindung zwischen dem (damals) letzten Buch der Autorin und ihrem ersten Roman hervorhebt: Der Fortschritt der Technik, der im 'Geteilten Himmel' bei der Nachricht von der ersten bemannten Raumfahrt als Triumph des wissenschaftlichen Geistes erscheint, wird im 'Störfall' bei der Meldung von der Katastrophe in Tschernobyl zur Bedrohung der Menschheit. Øhrgaard unterstreicht, daß

5 Das hat die Diskussion über ihre Erzählung 'Was bleibt' (1990) sehr deutlich gemacht. 
jene Nachricht und ihre Übermittlung in deutlicher Parallelität zur Weihnachtsgeschichte erzählt wird. Man könnte darauf hinweisen, daß diese naive Fortschrittsgläubigkeit allerdings schon in 'Nachdenken über Christa T.' (1968) ausdrücklich dementiert wird. ${ }^{6}$

Eine Vorlesung über "Wilhelm Tell und Niels Ebbesen - oder Wozu brauchen wir Nationalhelden in einem vereinten Europa?" ist für SvenAage Jørgensen Anlaß zu kritischer Selbstreflexion. Er zeigt zunächst, daß es zu Wilhelm Tell eine Parallele in der dänischen Geschichte gibt: Wie Tell, so befreite Niels Ebbesen im 14. Jahrhundert sein Land von einer fremden - norddeutschen - Übermacht, indem er deren Vertreter, einen holsteinischen Grafen, ermordete. Auch in Dänemark wird eine solche Begebenheit in der geschichtlichen Überlieferung zum Mythos - das kleine Land wird vom mächtigen Nachbarn überfallen, gedemütigt und ausgelaugt, bis ein Mann des einfachen Volkes aufsteht und durch seine Verzweiflungstat die Befreiung einleitet. Jørgensen bringt Beispiele für das Weiterwirken des Mythos bis ins 20. Jahrhundert und hebt hervor, daß Elemente dieses Mythos nach dem 2. Weltkrieg in der Schweiz wie in Dänemark dazu dienten, das Verhältnis des eigenen Landes zum nationalsozialistischen Deutschland in einem erträglichen Licht erscheinen zu lassen. Während aber Dürrenmatt und Frisch den Mißbrauch des nationalen Mythos anprangern und Frisch ihn mit seinem Büchlein 'Wilhelm Tell für die Schule' (1971) nachdrücklich demontiert, fehlt etwas Entsprechendes für den dänischen Schulgebrauch. Jørgensen bedauert dies ausdrücklich, wenn er Elemente dieses Mythos in den Argumenten der dänischen Gegner der EG aufspürt. Der Autor beschränkt sich auf solche Mythen, die in der Gestalt von Nationalhelden personifiziert sind. Eine Auseinandersetzung mit der grundsätzlichen Bedeutung von Mythen für das Selbstverständnis einer Nation hätte wohl den Rahmen einer Vorlesung gesprengt. ${ }^{7}$

Sven-Aage Jørgensens anderer Beitrag - "Die Deutschen und wir” schließt zeitlich an den von Vibeke Winge an und bietet einen Abriß über die Entwicklung des dänisch-deutschen Verhältnisses in den letzten 200

\footnotetext{
6 Das geschieht ebenfalls mit Hilfe biblischer Symbole:

"Frieden war plötzlich ein Wort, das gelten sollte, Vernunft, dachten wir, Wissenschaft: das wissenschaftliche Zeitalter. Da traten wir nachts auf den Balkon, um für Minuten eine Spur der neuen Sterne den Horizont entlangziehen zu sehen. ... Die glückliche, allen Anfängen günstige Zeit früher Unbefangenheit war vertan, wir wußten es. Wir schütteten den letzten Wein in den Apfelbaum. Der neue Stern hatte sich nicht gezeigt. Wir froren und gingen ins Zimmer ..."

(Wolf, Christa: Nachdenken über Christa T., Darmstadt 1971, S. 180 f. In späteren Auflagen S. 139).

7 Vgl. dazu neuerdings: Wülfing, Wulf u.a.: Historische Mythologie der Deutschen, München: Wilhelm Fink 1990.
} 
Jahren, hauptsächlich unter einem kulturellen Gesichtspunkt. Für das 18. Jahrhundert spricht der Autor von einem friedlichen Nebeneinander; Klopstock, der sich auf Einladung des dänischen Königs zwischen 1751 und 1770 die meiste Zeit in Kopenhagen aufhielt und dort eine Gruppe empfindsamer Intellektueller um sich sammelte, ist das berühmteste Beispiel. Noch Herder bezeichnete Kopenhagen als "das dänische Ende Deutschlands" (S. 148 u.ö.), Ausdruck nicht einer staatlichen Zugehörigkeit, sondern einer sprachlichen und kulturellen Gemeinschaft, die man in der gemeinsamen germanischen Vergangenheit verwurzelt sah. Das erwachende Selbstbewußtsein des Kopenhagener Bürgertums, das seinen Platz in der von Deutschen beherrschten staatlichen Führungsschicht beanspruchte, führte zwar gegen Ende des 18. Jahrhunderts zu Spannungen, aber dadurch wurde das deutschsprachige Kulturleben der Stadt nicht wesentlich beeinträchtigt. Es fiel erst den nationalliberalen Bewegungen des 19. Jahrhunderts zum Opfer, die eine Konfrontation mit der gesamten deutschen Kultur suchten. Vor allem Grundtvig rief zum Widerstand gegen das auf, was - auch im 20. Jahrhundert - als politischer und kultureller Imperialismus Deutschlands aufgefaßt wurde. Eine Gegenposition, die zu kritischer Selbstbesinnung auf die eigenen Werte aufforderte und u.a. von Kierkegaard formuliert wurde, konnte sich demgegenüber nicht durchsetzen. Der Widerstand führte nach den Ereignissen von 1848 bis 1864 dazu, daß sich Dänemark auch kulturell von Deutschland abkapselte. Jørgensen macht darauf aufmerksam, daß die damals entstandene Furcht vor dem übermächtigen Nachbarn, die durch die Besatzungszeit während des 2. Weltkrieges bestätigt und verstärkt wurde, bis heute nachwirkt und auch die Haltung vieler Dänen gegenüber Europa beeinflußt.

Das Buch versucht nicht, die im Buchtitel gestellte Frage nach den vier Kulturen zu beantworten, sie wird eigentlich in keinem der Beiträge diskutiert. Übrigens trägt die Karte auf dem Buchumschlag nicht unbedingt dazu bei, die mit dem Titel angesprochene Problematik zu veranschaulichen, denn dort erscheinen auch Luxemburg und Liechtenstein als deutschsprachige Staaten, was bei Luxemburg problematisch sein dürfte. Der Band verdeutlicht jedoch wesentliche Aspekte der dänischen Sicht auf das Verhältnis zum deutschen Nachbarn, dem einzigen Land, mit dem Dänemark eine gemeinsame Grenze hat. 
202 